[0212-7199 (2007) 24: 12; pp. 599-601] ANALES DE MEDICINA INTERN Copyright (C) 2007 ARAN EDICIONES, S.L.

AN. MED. INTERNA (Madrid) Vol. 24, N. ${ }^{\circ} 12$, pp. 599-601, 2007

\section{Varón de 69 años con afectación del estado general, hiperpigmentación, lesión cutánea y respuesta deficiente al tratamiento}

\author{
J. M. PRIETO DE PAULA, M. A. ALIAGA Y MONTILLA, J. I. ALONSO \\ FERNÁNDEZ, J. I. MARTÍN SERRADILLA, A. RELEA SARABIA ${ }^{1}$, \\ V. VILLAMANDOS NICÁS ${ }^{2}$
}

Servicios de Medicina Interna y de ${ }^{1}$ Radiodiagnóstico. Complejo Hospitalario de Palencia. ${ }^{2}$ Servicio de Análisis Clínicos. Hospital de Aranda de Duero. Burgos

69 YEARS OLD MALE PATIENT, WITH IMPAIRMENT OF PHYSICAL CONDITION, HYPERPIGMENTATION, CUTANEOS LESION AND A DEFICIENT RESPONSE TO TREATMENT

\begin{abstract}
RESUMEN
Presentamos el caso de un varón con insuficiencia suprarrenal primaria de etiología tuberculosa, con una lesión dérmica en la que se comprobaron granulomas necrotizantes y en cuyo cultivo creció micobacterium bovis. Dados los hallazgos clínicos, y a la espera de recibir el cultivo de la lesión, se inició tratamiento tuberculostático triple, que hubo de suspenderse por toxicidad hepática. Tras conocer el cultivo de $M$. bovis en la biopsia cutánea, se reinstauró el tratamiento con estreptomicina, rifampicina y etambutol. Tres semanas después, y a pesar de aumentar a $40 \mathrm{mg}$ diarios la dosis de hidrocortisona, reapareció la insuficiencia suprarrenal. Ante esta situación, se optó por mantener la rifampicina y duplicar la dosis de hidrocortisona, con lo que desparecieron totalmente las alteraciones clínicas y analíticas.

El interés del caso radica en la confluencia de tres procesos infrecuentes en la actualidad: la insuficiencia suprarrenal de etiología tuberculosa, la tuberculosis cutánea por micobacterium bovis y la insuficiencia suprarrenal primaria desencadenada por el uso de rifampicina, y resuelta con el incremento de dosis de hidrocortisona.
\end{abstract}

PALABRAS CLAVE: Insuficiencia suprarrenal primaria. Tuberculosis cutánea. Tuberculosis adrenal. Rifampicina. Crisis addisoniana.

\begin{abstract}
We submit the case of a male patient, suffering from a tuberculous ethiology adrenal primary insufficiency, showing a dermal lesion, in which necrotizing granulomas were found, and from which bacterial culture growth yielded mycobacterium bovis. Given the clinical findings, and awaiting for the bacterial culture result, a triple treatment with tuberculostatics was started, but had to be discontinued because of hepatic toxicity. After culture of cutaneous biopsy yielded micobaterium tuberculosis, treatment with streptomycin, rifampicin and etambutol was restarted. Three weeks later, in spite of increasing hydrocortisone dose to $40 \mathrm{mg}$, adrenal insufficiency reappeared. Under the circumstances, we chose to continue rifampicin and double hydrocortisone dose. The case is of concern because of the concurrency of three nowadays infrequent disorders: tuberculous ethiology adrenal insufficiency, cutaneous tuberculosis due to mycobacterium bovis and primary adrenal insufficiency due to rifampicin treatment, the latter resolved after increasing hydrocortisone dose.
\end{abstract}

KEY WORDS: Adrenal insufficiency primary. Cutaneous tuberculosis. Adrenal tuberculosis. Rifampicin. Addisonian crisis.

Prieto de Paula JM, Aliaga y Montilla MA, Alonso Fernández JI, Martín Serradilla JI, Relea Sarabia A, Villamandos Nicás V. Varón de 69 años con afectación del estado general, hiperpigmentación, lesión cutánea y respuesta deficiente al tratamiento. An Med Interna (Madrid) 2007; 24: 599-601.

\section{INTRODUCCIÓN}

La enfermedad de Addison o insuficiencia suprarrenal primaria (ISP) es un proceso poco frecuente, con una prevalencia en torno a 9-14 casos por 100.000 habitantes, cuya causa más frecuente en el mundo occidental y en la actualidad es la adrenalitis autoinmune.

La tuberculosis adrenal no supera, en nuestro medio, el $30 \%$ de los casos, y suele diagnosticarse ante la coexistencia de ISP con tuberculosis activa en otra localización, ante una prueba de Mantoux claramente positiva o ante unas suprarrenales agrandadas con calcio, en los casos de evolu- ción crónica (1). Otras causas más raras de ISP se exponen en la tabla I. Cabe insistir, por el caso que nos ocupa, sobre el hecho de que ciertos fármacos, entre los que se encuentra la rifampicina, pueden provocar insuficiencia suprarrenal.

La tuberculosis cutánea, por su parte, es una forma infrecuente de tuberculosis extrapulmonar -menos del $0,5 \%$ del total de casos de tuberculosis-, producida por Micobacterium tuberculosis, Micobacterium bovis, bacilo de Calmette Guérin y Micobacterium africanum (2), cuyas manifestaciones clínicas dependen tanto de la vía de infección como de la inmunidad del paciente frente al germen (3).

Trabajo aceptado: 30 de julio de 2007 
TABLA I

ETIOLOGÍA DE LA INSUFICIENCIA SUPRARRENAL PRIMARIA

Adrenalitis autoinmune

Aislada

Síndromes pluriglandulares autoinmunes I y II

Adrenalitis tuberculosa

Otras causas

Infecciones por hongos

$\mathrm{VIH}^{*}$ (citomegalovirus, criptococo, toxoplasma...)

Hemorragia suprarrenal (sepsis, coagulopatías, síndrome de Waterhouse-Friderichsen, tratamiento anticoagulante, anticuerpos antifosfolípido...)

Metástasis (mama, melanoma, pulmón, linfoma...)

Fármacos (ketoconazol, rifampicina, metopirona, aminoglutetimida, mitotano...)

Sarcoidosis

Amiloidosis

Deficiencias enzimáticas suprarrenales (hiperplasia suprarrenal congénita)

Adrenoleucodistrofia y adrenomieloneuropatía

Resistencia familiar a los glucocorticoides

Falta de respuesta congénita a los glucocorticoides Suprarrenalectomía bilateral

$\left({ }^{*}\right) \mathrm{VIH}$ : virus de la inmunodeficiencia humana.

Describimos a continuación el caso de un paciente con insuficiencia suprarrenal primaria de etiología tuberculosa y tuberculosis cutánea por micobacterium bovis, en el que -tras mejoría inicial con tratamiento sustitutivo- asistimos a un nuevo episodio de insuficiencia suprarrenal motivado por la rifampicina.

\section{CASO APORTADO}

Varón de 69 años de edad sin antecedentes de especial interés, que ingresa por astenia, pérdida de $10 \mathrm{~kg}$ de peso, cefalea, diarrea y vómitos ocasionales, todo ello de 3 meses de evolución.

En la exploración física destacaba una tensión arterial de 85/55 $\mathrm{mmHg}$, hiperpigmentación difusa, más intensa en codos, nudillos de manos, areolas mamarias y pliegues interdigitales, y una lesión indurada en la cara, de aspecto nodular y ligeramente eritematosa (Fig. 1).

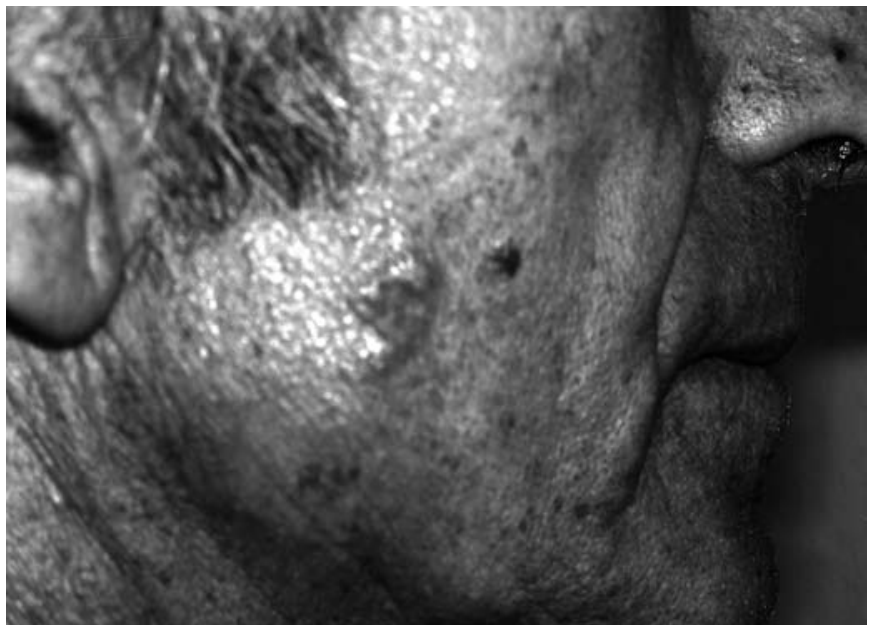

Fig. 1. Lesión nodular eritematosa en cara.
El sistemático de sangre y la bioquímica fueron normales, con excepción de una natremia de $129 \mathrm{mmol} / \mathrm{l}$ con natriuresis de 87 $\mathrm{mmol} / \mathrm{l}$. La radiografía de tórax también fue normal.

El test de estimulación con tetracosáctido reveló un cortisol basal de $7 \mu \mathrm{g} / \mathrm{dl}$; a los 30 minutos $6,22 \mu \mathrm{g} / \mathrm{dl}$; y a los 60 minutos $5,75 \mu \mathrm{g} / \mathrm{dl}$. Otros datos relevantes fueron: PPD $2 \mathrm{UT}$ : $9 \mathrm{~mm}$ de pápula a las 72 horas, anticuerpos antiadrenales negativos y ACTH: $398 \mathrm{pg} / \mathrm{ml}$-valor normal de 9 a 52-. La TSH, la serología frente al VIH, y los cultivos de esputo y de orina, en medio de Lowenstein, fueron normales o negativos. La biopsia de la lesión dérmica mostró granulomas necrotizantes, y en el cultivo creció Mycobacterium bovis.

En la TC abdominal se apreció una masa de $5 \times 3,2 \mathrm{~cm}$. en la glándula suprarrenal derecha, y un agrandamiento difuso de la glándula izquierda, con morfología conservada. Tras contraste intravenoso (Fig. 2), la lesión derecha presentaba un fino y homogéneo borde hipercaptante, con un área central también homogénea, sin captación, sugestiva de necrosis. Como hallazgos complementarios, se observaron adenopatías calcificadas mesentéricas, y pequeñas imágenes nodulares no calcificadas, de unos $8 \mathrm{~mm}$ de diámetro, adyacentes a la raíz del tronco celiaco, y en el retroperitoneo, también compatibles con adenopatías.

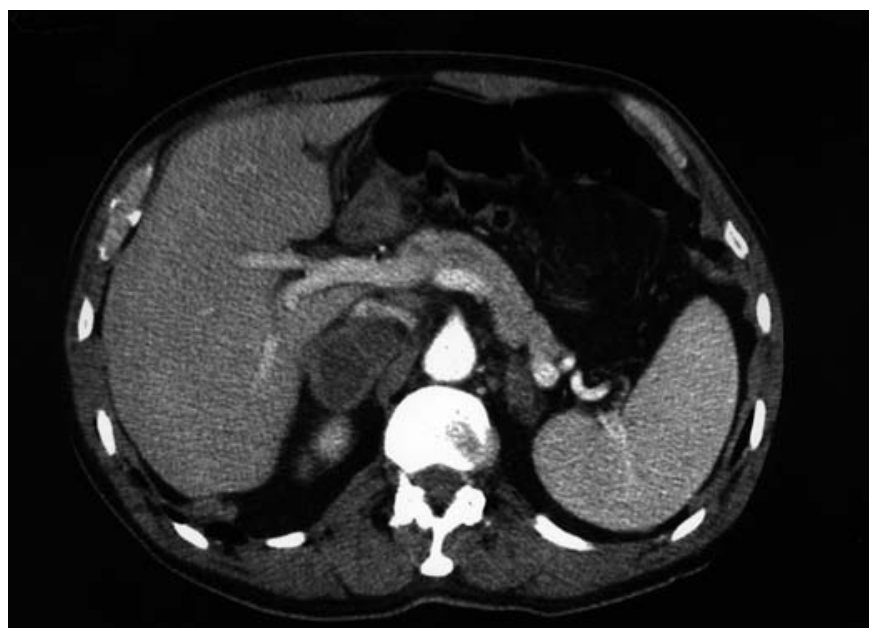

Fig. 2. Tras introducción de contraste, en fase portal, se aprecia un realce periférico, fino y homogéneo, con un centro hipodenso, que no capta, también homogéneo.

La punción de la suprarrenal derecha permitió extraer un líquido prácticamente acelular, con citología negativa para células tumorales, y cultivo negativo para micobacterias.

Tras conocer la presencia de granulomas caseificantes en la lesión dérmica, y a la espera de los cultivos, se inició tratamiento con $450 \mathrm{mg}$ de isoniacida, $900 \mathrm{mg}$ de rifampicina y $2.400 \mathrm{mg}$ de pirazinamida diarios. De igual modo, se pautó tratamiento sustitutivo con $30 \mathrm{mg}$ de hidrocortisona y $0,05 \mathrm{mg}$ de fludrocortisona. Tras una mejoría inicial llamativa, presentó una elevación de la ALT (420 UI/l) sin colestasis, que obligó a retirar los tuberculostáticos. A los 14 días, tras constatar normalización analítica, se reintrodujeron los tres fármacos, asistiendo a una nueva elevación de la ALT de 200 UI/l, que nos obligó a una nueva retirada. Fue en este período cuando conocimos el crecimiento de $M$. bovis en la biopsia cutánea, por lo que reinstauramos el tratamiento con estreptomicina, rifampicina y etambutol. Tres semanas después, y a pesar de incrementar la dosis de hidrocortisona a $40 \mathrm{mg} /$ día, apareció nuevamente astenia, hipotensión, hiponatremia $(129 \mathrm{mmol} / \mathrm{l})$ y elevación llamativa de la ACTH sérica $-1537 \mathrm{pg} / \mathrm{ml}$-. Ante esta situación, optamos por mantener los tuberculostáticos y duplicar la dosis de hidrocortisona, con lo que desparecieron totalmente las alteraciones clínicas y analíticas. 
Dos meses después, con $60 \mathrm{mg} /$ día de hidrocortisona, etambutol y rifampicina, el paciente se encuentra asintomático, con valores tensionales y natremia normales, y con desaparición de la lesión cutánea.

\section{DISCUSIÓN}

Como ya hemos expuesto, la enfermedad de Addison es un proceso poco frecuente que, en nuestro medio, es debida mayoritariamente a una adrenalitis autoinmune, bien aislada $o$ en el seno de un síndrome poliglandular. La tuberculosis adrenal es actualmente una causa infrecuente de ISP, que suele diagnosticarse ante la presencia de una tuberculosis activa en otra localización, ante un test tuberculínico claramente positivo o ante unas suprarrenales agrandadas con calcio.

La rifampicina es un potente inductor enzimático que incrementa la actividad del citocromo P450 y, en consecuencia, la oxidación del cortisol a 6- $\beta$-hidroxicortisol, un metabolito inactivo de aquél (4). Por tal motivo -y como ilustra el presente caso- es una causa reconocida de ISP.

La enfermedad de Addison se caracteriza clínicamente por la presencia de astenia, hiperpigmentación cutánea, pérdida de peso, manifestaciones gastrointestinales e hipotensión arterial.

Una vez planteada la sospecha, el diagnóstico se realiza mediante el test de estimulación rápida con $250 \mu \mathrm{g}$ i.v. de tetracosáctido, determinándose el cortisol antes, a los 30 y a los 60 minutos tras su administración. Un incremento del cortisol inferior al doble del basal, o que alcance cifras inferiores a $15 \mu \mathrm{g} / \mathrm{dl}$ se considera, en general, indicativo de ISP. Las concentraciones basales de ACTH, por otra parte, se encuentran elevadas.

El diagnóstico etiológico suele hacerse mediante el estudio de los anticuerpos antiadrenales, determinación muy sensible y cuya especificidad se acerca al $100 \%$. De igual modo, hay que valorar la presencia de infección o enfermedad tuberculosa, infecciones micóticas y enfermedad neoplásica. La tomografía axial computarizada es, en la actualidad, una técnica útil para conocer la etiología de la enfermedad de Addison, aunque sus hallazgos pueden variar en función tanto de la causa como del estadio evolutivo de la enfermedad. Así, en la fase aguda de las infecciones, se puede observar aumento del tamaño de las suprarrenales, generalmente con necrosis central y realce periférico; mientras que en las fases muy evolu-

\section{Bibliografía}

1. Barceló Lucerga B. Enfermedades de la corteza suprarrenal. En: Rodés Teixidor J, Guardia Massó J, editores. Medicina Interna. Barcelona. Ediciones Masson S.A., 2004; 2492-2513.

2. Carapeto FJ. Tuberculosis cutánea. ¿Enfermedad emergente? Piel 2004; 19: 63-66.

3. Ramos Rincón JM, Fernández Guerrero ML. Tuberculosis extrapulmonar en pacientes sin infección por el virus de la inmunodeficiencia humana. Revisión de aspectos clínicos relevantes. Rev Clin Esp 1996; 196: 39-48.

4. Kyriazopoulou V, Vagenakis AG. Abnormal overnight dexamethaxone supresión test in subjects receiving rifampicin therapy. J Clin Endocrinol Metab 1992; 75: 315-317.

5. Bacarizo P, Checa MR, Suárez Llanos P, Hernández L, Jara Albarrán A. Evolución radiológica en el seguimiento de la adrenalitas tuberculosa. Endocrinol Nutr 2006; 53: 510-514

6. Halperin Rabinovich I. Diagnóstico de la enfermedad de Addison. Clí- cionadas, las glándulas pueden estar atróficas y presentar calcificaciones; lo cual sugiere afectación tuberculosa (5). Estos hallazgos contrastan con la disminución de tamaño observada en la adrenalitis autoinmune y con la afectación metastásica de las suprarrenales, donde también se produce aumento de su tamaño y, a menudo, configuración alterada y borramiento de los límites con las estructuras vecinas. La PAAF estaría indicada ante aumentos de tamaño de la glándula, con o sin calcificaciones, a menos de que existan datos fehacientes de tuberculosis activa -como ocurría en el presente caso-, o de metástasis de una neoplasia conocida (6).

El tratamiento de la ISP consiste en la administración de hidrocortisona a dosis aproximada de $30 \mathrm{mg} /$ día. Dado que, además, tiene efecto mineralocorticoide, puede utilizarse o no -de acuerdo con la tensión arterial, el $\mathrm{Na}$ y el $\mathrm{K}$ séricos- fludrocortisona a dosis de 0,05-1 mg/día (7).

La tuberculosis cutánea es una forma rara de tuberculosis extrapulmonar, producida por $M$. tuberculosis, $M$. bovis, el bacilo de Calmette Guérin y M. Africanum (2), cuyas manifestaciones clínicas dependen de la vía de infección y de la inmunidad del paciente frente al germen.

El lupus vulgar - la variedad del presente caso- es la forma más habitual de tuberculosis cutánea en los países desarrollados, incluido España. Se produce por diseminación hematógena, y se caracteriza por la aparición de una lesión única, asintomática y progresiva, formada por nódulos eritemato-violáceos que pueden evolucionar a ulceraciones o cicatrices, y que se localiza preferentemente en la cara. El diagnóstico suele realizarse ante la presencia de granulomas necrotizantes y el crecimiento del germen en el medio de Lowenstein, ya que la baciloscopia solamente es positiva en el $6 \%$ de los casos. La técnica de la PCR ha demostrado su utilidad en el diagnóstico, pero puede ser negativa en las formas paucibacilares (8). El tratamiento es similar al de otras formas de tuberculosis. Cabe citar el matiz de la resistencia constitutiva de $M$. bovis a la pirazinamida, debido a una mutación en el codón 169, en el gen pncA (9).

Resulta relevante, para finalizar, la reaparición de la insuficiencia suprarrenal unas semanas después del inicio del tratamiento con rifampicina, y cuya causa analizamos más arriba. Aunque la recomendación habitual es la retirada del fármaco, nuestro caso se resolvió con un incremento de la dosis de hidrocortisona -tal y como aconsejan otros autores-, en tanto persista el uso de la rifampicina $(10,11)$. nica, radiología, análisis y biopsia. ¿Todos para uno o uno para todos? Med Clin (Barc) 1993; 101: 132-135.

7. Alonso N, Granada ML, Lucas A, Salinas I, Sanmartí A. Enfermedad de Addison. Tratamiento sustitutivo con glucocorticoides y su monitorización. Endocrinol Nutr 2003; 50: 390-395.

8. Arora SK, Kumar B, Sehgal S. Development of a polymerase Chain reaction dot-blotting system for detecting cutaneous tuberculosis. Br J Dermatol 2000; 142:72-76.

9. Coll P. Fármacos con actividad frente a Mycobacterium tuberculosis. Enferm Infecc Microbiol Clin 2003; 21: 299-308.

10. Wilkins EG, Hnizdo E, Cope A. Addisonian crisis induced by treatment with rifampicin. Tubercle 1989; 70: 69-73.

11. Lorente R, Ampudia-Blasco FJ, Catalá M, Carmena R. Insuficiencia suprarrenal aguda inducida por rifampicina. Rev Clin Esp 1997; 197: 725-726 